WWW.iibpas.com

\title{
A CASE REPORT OF OSTEOPHYTIC CHANGES IN THE CADAVER
}

\section{SHARMA $\mathrm{M}^{1^{*}}$, MESHRAM AA ${ }^{2}$, PATEL NS ${ }^{3}$ AND CHAUHAN A}

1: Final Year PG Scholar, Dept. of Rachana Sharir, Parul Institute of Ayurveda, Parul University

2: Professor, Dept. of Rachana Sharir, Parul Institute of Ayurveda, Parul University

3: Asst. Professor, Dept. of Rachana Sharir, Parul Institute of Ayurveda, Parul University

4: Final Year PG Scholar, Dept. of Rachana Sharir, Parul Institute of Ayurveda, Parul University

*Corresponding Author: Dr. Maneela Sharma: E Mail: maneelasharma12@gmail.com

Received 12 ${ }^{\text {th }}$ Dec. 2021; Revised $14^{\text {th }}$ Jan. 2022; Accepted $7^{\text {th }}$ Feb. 2022; Available online $5^{\text {th }}$ March 2022

https://doi.org/10.31032/IJBPAS/2022/11.3.1097

\begin{abstract}
The most prevalent form of arthritis, osteoarthritis (OA), is now known to affect all joint tissues, including active anabolic and catabolic processes. Knee OA, in particular, is thought to be primarily a mechanical illness. Bone changes are expected to have a role in the development of OA because bone adjusts to stresses by remodelling to fulfil its mechanical demands. Changes in subchondral bone turnover, mineralization, and volume result in changed apparent and material bone density, which may have an unfavourable effect on the biomechanical environment of the joint. One of the symptoms of osteoarthritis is an osteophyte, which is a fibrocartilage-capped bony outgrowth.

On a female cadaver, we offer a case study of osteoarthritis with osteophytic alterations. During a regular dissection at Vadodara's Parul Institute of Ayurveda (Gujrat). On the left femur, we found severe osteophytic alterations (Figure $1 \& 2$ ), and on the right knee joint, we noticed a prosthesis of Total Knee Arthroplasty/ Knee Replacement Surgery. It was discovered in a 68 -year-old female corpse with an over-weighted body build.
\end{abstract}

Keywords: Osteoarthritis, osteophytic, cadaver, knee joint 


\section{INTRODUCTION}

Knee osteoarthritis (OA) is a frequent, painful, and debilitating disorder. It affects up to $30 \%$ of the population in Western countries over the age of 651. Degenerative arthritis can be a complex disease. Much earlier research looked at the patterns of OA and attempted to categorize subgroups based on the anatomical location of component markings found on x-rays'2. Changes evident on radiography, as well as constriction at the joint region, osteophytes, subchondral bone sclerosis, and cysts 3 , are used by clinicians to identify degenerative arthritis. One of the distinguishing features of osteoarthritis is the presence of an osteophyte4. Osteophytes are the most common anomaly associated with the persistent joint deformity. Osteophytes in the femur head and neck, as well as uneven joint region constriction, subchondral cysts, and bony sclerosis, are common in individuals with degenerative arthritis.

\section{LITERATURE REVIEW}

\section{FEMUR}

The femur is the strongest and longest bone in the human body. The femoris articulates with the hip bone proximally. It interacts with the patella and the proximal facet of the tibia5 from a distance. During the 5 th to 6th week of pregnancy, the femur begins to grow by endochondral ossification. Ossification of the femoris is finished between the 14th and 18th years of life6, but several ossification foci appear during intrauterine life. The existence of the medial and lateral condyles, which articulate with the shinbone and patella to form the complicated knee joint, distinguishes the femur distally.

Medial and lateral condyles - The femur is the strongest and longest bone in the human body. The femoris articulates with the hip bone proximally. It interacts with the patella and the proximal facet of the tibia5 from a distance. During the 5th to 6 th week of pregnancy, the femur begins to grow by endochondral ossification. Ossification of the femoris is finished between the 14th and 18th years of life6, but several ossification foci appear during intrauterine life. The existence of the medial and lateral condyles, which articulate with the shinbone and patella to form the complicated knee joint, distinguishes the femur distally.

Medial and lateral epicondyles - Nonarticular portions of the condyles have bony elevations. The medial epicondyle is bigger than the lateral epicondyle. The epicondyles of the medial and lateral collateral ligaments of the knee are the origins of their respective ligaments9.

Intercondylar fossa - Between the two condyles on the posterior surface of the 
femur, there is a deep notch. It has two aspects for intracapsular knee ligament attachment; the anterior cruciate ligament (ACL) connects to the medial aspect of the lateral condyle, while the posterior cruciate ligament (PCL) connects to the lateral aspect of the medial condyle 10 .

\section{OSTEOPHYTES}

Bony excrescences, known as osteophytes, are the product of one of two types of bone creation: endochondral bone formation, in which cartilages lay down bone, or intramembranous bone formation, in which the periosteal membrane generates bone. Osteophytes form as a result of vascular invasion and erosion of the subchondral bone plate and calcified cartilage, with deposition of on the worn surface. As a result, they're most noticeable in areas of the articulation that are subjected to low stress; at these sites, parts of the cartilaginous articular coat are still present, requiring endochondral bone formation. As a result, the largest osteophytes are often seen on the margins or at the periphery of the distribution. The extension of bone burys a portion of the initial zone of calcified cartilages as it expands, which may be seen on radiographs and pathologic examination. Osteophytes might also grow as a result of intramembranous bone development, which occurs when the synovial membrane, the intraarticular counterpart of the periosteal membrane, is disturbed to supply new bony tissue.

\section{CLASSIFICATION}

Extraspinal osteophytes are those that grow outside of the spine. There are four types of extraspinal osteophytes, according to their radiographic studies and creation mechanism11. Marginal osteophytes appear as a 'lip' at the joint's outer edge. And are generated by endochondral ossification promoted by subchondral bone marrow vascularization 12 . They are present in nonpressure segments and are hence not always associated with significant surrounding disease or subchondral cysts. They usually concentrate on one side of the joint. Central osteophytes are protrusion from the interior joint region with a rough articular surface 13. Osteophytes of the periosteum and synovium appear as thickened intraarticular cortices. Capsular osteophytes grow in the direction of capsular tension as a result of capsular traction14. Traction or claw 15 osteophytes are two types of spinal osteophytes.

\section{PREVELENCE}

Symptomatic knee osteoarthritis affects around $13 \%$ of women and $10 \%$ of men aged 60 and over. The percentage of persons with symptomatic knee osteoarthritis are predicted to rise as the population ages and the overall population's obesity or overweight rate rises 16. 
Females, especially those over 55, had more severe osteoarthritis in the knee than men, but not in other joints. The findings of this investigation revealed a sex difference in the incidence of knee osteoarthritis, especially after menopause 17 .

\section{ETIOLOGY AND RISK FACTORS}

Degenerative osteoarthritis is the most prevalent cause of bone spurs. This is the type of inflammatory illness brought on by joint wear and tear over time. Degenerative arthritis is more common in older persons, although it can start sooner if a joint has been fractured due to a sports injury, an accident, or another reason. The cartilages at the ends of bones wear down in a joint with inflammatory illness, such as arthritis. The elastic tissue that links and cushions the bones of a joint is known as cartilage. New bone material emerges within the variety of bone spurs as part of the body's effort to mend the torn cartilages. Osteoarthritis has a complicated aetiology that results from the interaction of local and systemic causes. Arthritis degenerative affects people of all ages. The genesis of this severe condition, in which numerous genes are implicated in its occurrence. Adolescent athletes are predisposed to early degenerative arthritis due to sports activity, joint damage, obesity, and hereditary vulnerability. Knee degenerative arthritis is 3.86 times more likely among people who have had previous knee trauma18. Age, female gender, obesity, knee injury, repetitive joint usage, bone density, muscular weakness, and joint neglect all play a part in the development of joint degenerative arthritis.

Risk factors of knee osteoarthritis;

1. Age

2. Genetic susceptibility

3. Obesity Female gender

4. Trauma

5. Repetitive knee trauma

6. Muscle weakness

7. Joint laxity

8. Mechanical forces

\section{Kneeling Squatting}

\section{Meniscal injuries}

\section{PROCEDURE}

Cunningham's dissection handbook was used to open the knee. We reflected the skin, superficial fascia, and deep fascia before cutting through the quadriceps tendon just proximal to the patella. At 2$3 \mathrm{~cm}$ on either side of the patellar tendon, the incision was prolonged downwards to the tibial condyles. The patella descended, exposing the knee hollow.

\section{CASE REPORT}

During a regular dissection of PGs at the Rachana Sharir department of the Parul Institute of Ayurveda in Vadodara (Gujrat). In the left knee joint, we discovered that the articular surface of the femur was 
significantly osteophyte, indicating aberrant bone development. A total knee arthroplasty was performed on the right knee, and a prosthesis was discovered. It was discovered in a corpse of a 68 -year-old adult female. Other than that, there were no noticeable abnormalities in the other limbs. Photographs were taken and pasted on this piece of paper.
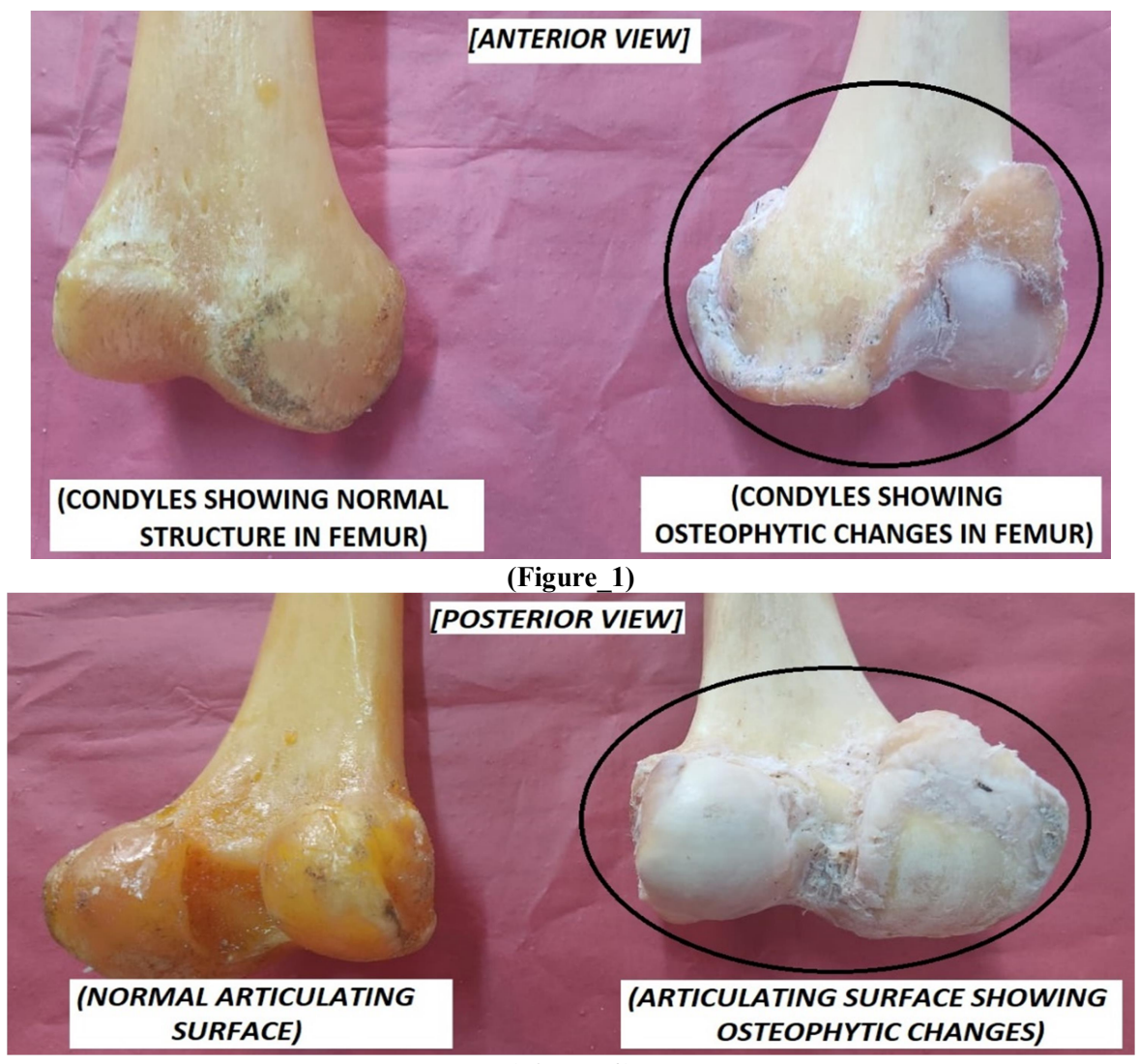

(Figure 2)

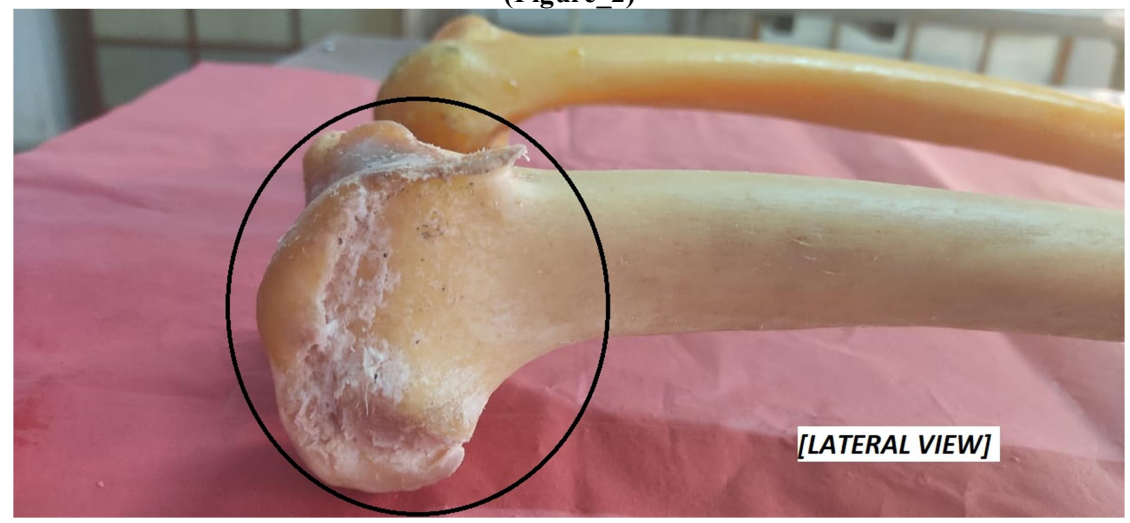

(Figure_3) 

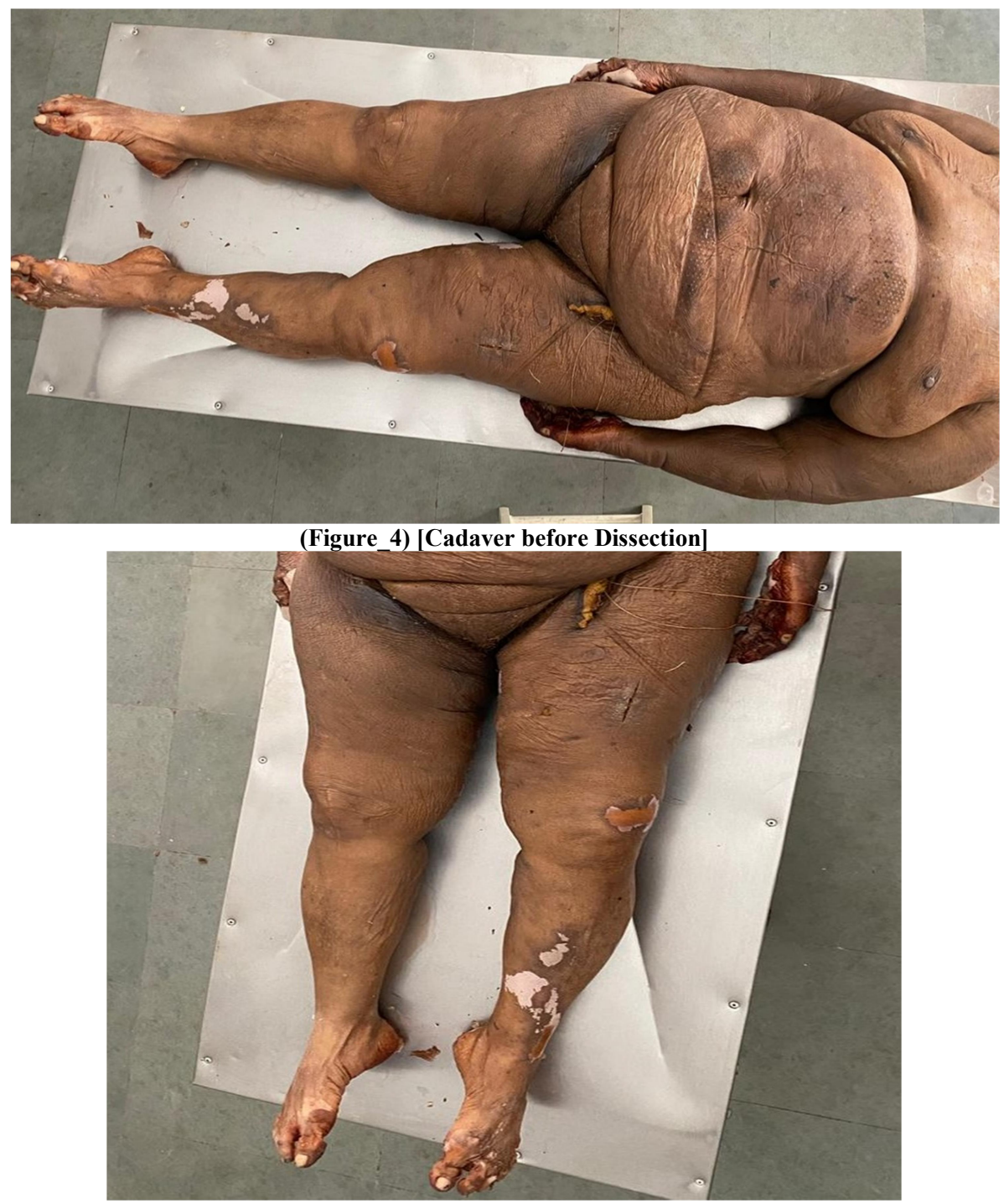

(Figure_4) [Intact Lower Limbs before dissection]

\section{DISCUSSION}

$\checkmark$ Osteophytes may develop as a result of joint instability, according to certain theories. If this is the case, the intercondylar osteophytes of the femur are caused by an area of instability. The intercondylar area is intricately connected with the cruciate ligaments. When the knee "screws home" near the conclusion of leg extension, they maintain tight knee stability and prevent the femur from sliding anteriorly in flexion and posteriorly in extension. A lack of or injury to these ligaments might result in knee instability affecting this region.

$\checkmark$ The knee joint, on the other hand, is intricate, impacting the function of multiple connected tissues such as the ligamentum patellae and suprapatellar bursa. The common popliteal artery, for example. 
Until recently, the presence of osteophytes was required for a radiographic-based definition of OA.

$\checkmark$ During OA, the subchondral bone structure changes dramatically, and it has long been considered that this is due to cartilage deterioration.

$\checkmark$ We should show kids such an atypical structure so they can understand the difference between normal and abnormal.

\section{CONCLUSION}

Based on the above observation and discussion it's concluded that on the left lower limb of the cadaver osteophytes found on the articular surfaces of the femur bone, which clear identification mark of the degenerative osteoarthritis, and additionally prosthetic device found on the right knee joint that's due to the overweighted

body weight and there's no the other reason seen for TKA surgery.

\section{ACKNOWLEDGEMENT}

The anatomical variation "A CASE OF OSTEOPHYTIC CHANGES ON A CADAVER" is noted by me, while conducting a routine dissection for Post Graduate study in Parul Institute of Ayurveda, Vadodara (Gujrat). I would like to pay my sincere gratitude to Dr. Hemant Toshikhane, Dean \& Principal, Dr. Bandapallae Dattu Narayanrao,
HOD \& Prof. Rachana Sharir Department, Dr. Akashdeep A Meshram, Prof. Rachana Sharir Department, Parul Institute of Ayurveda, Vadodara and Dr. Ajit S. Wahane, Associate Professor and Dr. Nirav S Patel and Dr. Kishan Singh, Asst. professor, Dept of Rachana Sharir, Parul Institute of Ayurveda, Parul University, Vadodara, Gujarat for valuable support.

\section{REFRENCES}

[1] van Saase JLCM, van Romunde LKJ, Cats A, Vanden broucke JP, Valkenburg HA. Epidemiology of osteoarthritis: Zoetermeer survey. Comparison of radiological osteoarthritis in a Dutch population with that in 10 other populations. Ann Rheum Dis 1989; 48:271-80.

[2] Distribution of distal femoral osteophytes in a human skeletal population Lee Shepstone, Juliet Rogers, John Kirwan, Bernard Silverman Ann Rheum Dis 2000;59:513-520 Ann Rheum Dis: first published as $10.1136 / \operatorname{ard} .59 .7 .513$ on 1 July 2000

[3] Kellgren JH, Lawrence JS. Radiological assessment of osteoarthrosis. Ann Rheum Dis 1957;16:494-502 
[4] Kellgren JH, Lawrence JS. Radiological assessment of osteo-arthrosis. Ann Rheum Dis 1957;16:494-502

[5] https://www.kenhub.com/en/library/ana tomy/femur

[6] https://teachmeanatomy.info/lowerlimb/bones/femur/

[7] https://www.kenhub.com/en/library/ana tomy/femur

[8] https://teachmeanatomy.info/lowerlimb/bones/femur/

[9] Resnick D. Degenerative disease of extraspinal locations. In: Resnick D, editor. Diagnosis of bone and joint disorders. Vol II, 4th ed. Philadelphia: Saunders; 2002:1287-9. https://www.kenhub.com/en/library/ana tomy/femur

[10]Resnick D. Degenerative disease of extraspinal locations. In: Resnick D, editor. Diagnosis of bone and joint disorders. Vol II, 4th ed. Philadelphia: Saunders; 2002:1287-9.

[11]Jaffe HL. Metabolic, degenerative and inflammatory diseases of bones and joints. Philadelphia: Lea \& Febiger; 1972.

[12] Siu Him Janus Wong, Kwong Yuen Chiu, Chun Hoi Yan Department of Orthopaedics \& Traumatology, The
University of Hong Kong, Hong Kong Journal of Orthopaedic Surgery 2016;24(3):403-10 Vol. 24 No. 3, December 2016

[13] Siu Him Janus Wong, Kwong Yuen Chiu, Chun Hoi Yan Department of Orthopaedics \& Traumatology, The University of Hong Kong, Hong Kong Journal of Orthopaedic Surgery 2016;24(3):403-10 Vol. 24 No. 3, December 2016

[14] Macnab I. The traction spur. An indicator of segmental instability. J Bone Joint Surg Am 1959;41:1047.

[15] Zhang Y, Jordan JM. Epidemiology of osteoarthritis. Clin Geriatr Med 2010; 26: 355- 69 .

[16] Behzad Heidari (MD), Knee osteoarthritis prevalence, risk factors, pathogenesis and features: Part I, Department of Internal Medicine, Rouhani Hospital, Babol University of Medical Sciences, Babol, Iran

[17] Blagojevic $M$, Jinks C, Jeffery A, Jordan KP. Risk factors for onset of osteoarthritis of the knee in older adults: a systematic review and meta analysis. Osteoarthritis Cartilage 2010; 18: 24- 33 . 